\title{
LACUNAS CIENTÍFICAS SOBRE GESTÃO DE RESÍDUOS: CONTRIBUIÇÃO DA LITERATURA PARA UM PLANETA MAIS SUSTENTÁVEL
}

\section{Rafaela Garbelini Anuardo, Maximilian Espuny, José Salvador da Motta Reis, Otávio José de Oliveira.}

\author{
Universidade Estadual Paulista /Faculdade de Engenharia de Guaratinguetá, Avenida Dr. Ariberto \\ Pereira da Cunha, 333, Bairro - 12244-000 - Guaratinguetá-SP, Brasil, r.anuardo@unesp.br, \\ maximilian.espuny@unesp.br, jmottareis@gmail.com, otavio.oliveira@unesp.br.
}

\begin{abstract}
Resumo - A geração de resíduos é consequência natural da urbanização, desenvolvimento econômico e crescimento populacional. Na medida em que as nações e as cidades se tornam mais populosas, oferecem mais produtos e serviços aos cidadãos e participam do comércio e do intercâmbio global, enfrentando grandes quantidades de resíduos para gerenciar por meio de tratamento e descarte. O objetivo deste trabalho é identificar as lacunas científicas existentes sobre o tema, o que servirá de guia para estudos futuros contribuindo para o avanço desse campo de conhecimento. A pesquisa será realizada a partir da análise de conteúdo dos 30 artigos científicos mais citados dos últimos cinco anos (2014 - 2019), relacionados a gestão de resíduos e disponíveis na base de dados Scopus. A principal contribuição deste trabalho foi a identificação dos grupos pesquisa: energia renovável, estruturas de coleta e reciclagem, estruturas de destinação final de resíduos; ferramentas e métodos para gestão de resíduos e práticas sustentáveis.
\end{abstract}

Palavras-chave: Gerenciamento de Resíduos, Análise de conteúdo, Lacunas Científica. Área do Conhecimento: Engenharias. Engenharia de Produção.

\section{Introdução}

A geração de resíduos é consequência natural da urbanização, desenvolvimento econômico e crescimento populacional. Na medida em que as nações e as cidades se tornam mais populosas, oferecem mais produtos e serviços aos cidadãos e participam do comércio e do intercâmbio global, enfrentando grandes quantidades de resíduos para gerenciar por meio de tratamento e descarte (WORLD BANK GROUP, 2018). O problema da gestão de resíduos não é novo (MILUTINOVIĆ et al., 2014). Na era industrial, a extração de recursos e a produção de bens têm se expandido constantemente para satisfazer o crescente consumismo. Uma gama de produtos como tecidos e produtos eletrônicos, antes tratados como itens de luxo, agora são usados como produtos do cotidiano. Como resultado, os resíduos produzidos hoje são de fontes mistas, prejudiciais ao meio ambiente e onerosos para serem geridos de forma sustentável (ZAMAN, 2015).

O desenvolvimento sustentável é uma das principais preocupações atuais na pauta dos estudos da gestão, organização e negócios. O desenvolvimento sustentável pode ser definido como um meio de atender às necessidades do presente sem comprometer a subsistência das gerações futuras. A dimensão ambiental, apesar de importante, é um componente do conceito de sustentabilidade que leva em consideração às dimensões social e econômicas, além da natural (CULLEN, 2017). Com a crescente produção de resíduos comprometendo o desenvolvimento sustentável verifica-se dificuldade acentuada na sua gestão em cidades de todo o mundo (DONG et al., 2014; NABAVIPELESARAEl et al., 2017). Governos e cidadãos têm se tornado conscientes e apreensivos sobre como os resíduos são gerenciados (NABAVI-PELESARAEl et al., 2017).

As práticas de gestão de resíduos evoluíram ao longo dos séculos. Enquanto no período inicial do gerenciamento havia uma preocupação maior com a higiene, nas sociedades de hoje a quantidade e a complexidade dos resíduos tornaram-se as principais questões da gestão de resíduos. Paralelamente ao desenvolvimento econômico, a gestão de resíduos passou por várias etapas para o aprimoramento tecnológico observado hoje. Sistemas de coleta sofisticados, combinados com processos de separação eficientes, permitem altas taxas de recuperação e reciclagem (BRUNNER; RECHBERGER, 2015). A quantidade de resíduos gerada em 2010 na União Europeia (UE) foi de 258 milhões de toneladas, nos EUA foi de 230 milhões de toneladas e na China foi de 154 milhões de toneladas (MILUTINOVIĆ et al., 2014). Além isso, a quantidade de resíduos gerados tem crescido 
aproximadamente 20\% em relação aos anos de 1995 a 2006, tanto na América do Norte quanto na UE (GHIANI et al., 2014).

Frente a esse aumento, o processo de tomada de decisão sobre a gestão de resíduos é uma questão complexa, que deve avaliar e considerar adequadamente os impactos ambientais, aspectos técnicos, implementação e custos operacionais de cada tratamento e disposição específicas e suas implicações sociais (ARENA; DI GREGORIO, 2014). Este trabalho terá o seu desenvolvimento por meio de uma análise de conteúdo sobre a gestão de resíduos, que tem como característica a apreciação de documentos críticos com o enfoque na definição das características de um determinado texto, buscando identificar elementos que permitam a compreensão de um estudo da atual e aplicá-lo a um tema proposto (HSIEH; SHANNON, 2005).

Estudos bibliométricos sobre resíduos sólidos têm sido conduzidos desde 2010. Fu et al. (2010) analisaram os períodos de 1993 a 2008 utilizando o banco de dados do Índice de Citação Científica (SCl). Comparado com estes estudos anteriores, este artigo atualiza e amplia a análise do estado da arte sobre Gestão de Resíduos Sólidos. Em função do exposto, a questão de pesquisa que norteará a realização desse trabalho é: quais são as principais lacunas científicas e tendências de pesquisa na gestão de resíduos para a contribuição de um planeta mais sustentável? O objetivo deste trabalho é identificar as lacunas científicas existentes sobre o tema, o que servirá de guia para estudos futuros contribuindo para o avanço desse campo de conhecimento.

\section{Metodologia}

A Tabela 1 apresenta a classificação desta pesquisa quanto a sua Natureza, Objetivos/Fins, Abordagem e Procedimentos Técnicos.

Tabela 1- Classificação da Pesquisa.

\begin{tabular}{ll}
\hline Natureza & Aplicada \\
Objetivo/fins & Descritiva \\
Abordagem & Qualitativa \\
Procedimentos Técnicos & Pesquisa bibliográfica e Análise de Conteúdo \\
\hline \multicolumn{2}{c}{ Fonte: Adaptado de Kothari \& Garg (2019). }
\end{tabular}

De acordo com o objetivo, essa pesquisa pode ser classificada como descritiva pois haverá a observação, o registro e análise dos dados coletados, sem manipulá-los, isto é, sem a interferência dos pesquisadores (KOTHARI; GARG, 2019).

Quanto a abordagem, essa pesquisa pode ser considerada qualitativa, pois realiza a interpretação e a atribuição de significados aos fatos em estudo (MIGUEL, 2018). Em relação aos procedimentos técnicos será realizada uma pesquisa bibliográfica e uma análise de conteúdo, que permitirá, por meio do processo de conhecimento e exame sistemático de uma certa realidade, observar um determinado conjunto, assim como perceber possíveis relações e formular ideias chave, levando a generalização e à possíveis críticas (KOTHARI; GARG, 2019). A coleta de dados foi realizada na base de dados Scopus no mês de abril de 2019. Foram selecionados na pesquisa somente aqueles publicados em inglês, por ser a língua mais comumente usada na ciência (NUNHES; OLIVEIRA, 2018).

A coleta de dados foi realizada na base de dados Scopus no mês de abril de 2019. Foi utilizado o descritor na pesquisa "Waste Management", optou-se somente por esse termo em palavras do título, para possibilitar pegar artigos com bastante aderência à proposta do artigo. Foram identificados na pesquisa 2199 estudos indexados. Para a identificação de lacunas científicas relacionadas a pesquisa foram utilizados os 30 artigos mais citados na base, considerando o recorte temporal de 2014 a 2019. Os dados foram tratados por meio do software Microsoft Excel.

\section{Resultados}

Nos principais resíduos que foram apresentados nas 30 publicações mais relevantes, identificouse os sólidos e sólidos urbanos, alimentares, radioativos, de construção e demolição, hospitalares e eletroeletrônicos, que foram apresentados principalmente pelos autores Ghiani et al. (2014), Sellin e Leupin (2013), Yay (2015), Gupta et al. (2015), Thi et al. (2015), Salemdeeb et al. (2015), Windfeld e Brooks (2015), Butera et al. (2015), Dahlbo et al. (2015), Sarath et al. (2017). 
Vale ressaltar que cada país tem uma regulamentação própria quanto à classificação dos resíduos. No caso do Brasil, a mais importante é a Lei no 12.305/10 que institui a Política Nacional de Resíduos Sólidos (PNRS). Vale destacar também a norma NBR 10004 (ASSOCIAÇÃO BRASILEIRA DE NORMAS TÉCNICAS, 2004), que estabelece um conjunto de padrões a ser utilizado pelas partes interessadas nas questões que envolvam os resíduos sólidos. Para esta norma a definição de resíduos sólidos é qualquer resíduo nos estados sólido ou semi-sólido, provenientes de atividades industriais, domésticas, hospitalares, comerciais e agrícolas, incluindo nesta definição os lodos provenientes de sistemas de tratamento de água.

Seguindo esse raciocínio, buscou-se pela a organização das lacunas dos artigos mais relevantes sobre o tema, para a realização da análise de cada agrupamentos, que pode ser identificada na Tabela 2. Os grupos de lacunas que se sobressaíram foram: Energia renovável; Estruturas de coleta e reciclagem; Estruturas de destinação final de resíduos; Ferramentas e métodos para gestão de resíduos e Práticas sustentáveis. A lacuna identificada no artigo com o maior número de citações foi a que continha o conteúdo "Realização de estudos sobre LCA aplicados a resíduos sólidos em países subdesenvolvidos e emergentes". A segunda lacuna advinda do artigo mais citado foi "Propor aplicações dos padrões ISO e das diretrizes do Manual do ILCD em resíduos sólidos". Em terceiro, "Desenvolvimento de estruturas de coleta voltadas às partes específicas de itens tecnológicos". A quarta lacuna foi "Aplicação da teoria dos jogos para conciliar interesses dos stakeholders no âmbito dos resíduos sólidos", e a quinta "Desenvolvimento de abordagens eficientes de integração dedicadas a estratégias de gestão de resíduo".

Tabela 2- Classificação da Pesquisa.

Tendências de Pesquisa

Energia renovável

Estruturas de coleta e reciclagem

Estruturas de destinação final de resíduos

Ferramentas e métodos para gestão de resíduos

Práticas sustentáveis
Autores

(AGHAJANI MIR et al., 2016; QAMBRANI et al., 2017)

(ALLESCH; BRUNNER, 2014; DAHLBO et al., 2015; ERIKSSON; STRID; HANSSON, 2015; ERSES YAY, 2015; RIGAMONTI et al., 2014; SARATH et al., 2015; WINDFELD; BROOKS, 2015) (SELLIN; LEUPIN, 2014; NABAVI-PELESARAEI et al., 2017; LIU et al., 2014; BUTERA et al. 2015)

(ALLESCH; BRUNNER, 2014; DONG et al., 2014; GHIANI et al., 2014; GUPTA; YADAV; KUMAR, 2015; KANAGARAJ et al., 2015; LAURENT et al., 2014a, 2014b; MIEZAH et al., 2015; SOLTANI et al., 2015; TAN et al., 2014; TATUM; FOY; APRAHAMIAN, 2014; WILSON et al., 2015)

(ARENA; DI GREGORIO, 2014; FERNÁNDEZ-NAVA et al., 2014; SALEMDEEB et al., 2017; THI; KUMAR; LIN, 2015; YONG et al., 2016)

Fonte: Elaborado pelos próprios autores (2020).

A principal tendência que apresentou um maior número de lacunas foi Ferramentas e métodos para gestão de resíduos, com 12 apontamentos, enquanto em Energia renovável, apresentou-se apenas duas.

\section{Discussão}

Em Energia Renovável, aborda-se a utilização de resíduos menos biodegradáveis para serem submetidos a produção de energia e a produção de carvão biodegradável em escala, diminuindo a toxicidade da queima das matrizes energéticas poluentes (AGHAJANI MIR et al., 2016; QAMBRANI et al., 2017).

Quanto às Estruturas de coleta e reciclagem, identificam-se estudos que são mais voltados equipamentos tecnológicos, resíduos orgânicos e estudos com características mais generalistas. Brunner e Rechberger (2014) e Sarath et al. (2015), mencionam a importância de se estruturar locais que possam receber equipamentos de tecnologia, diminuindo os riscos ambientais. Eriksson, Strid e Hansson (2015) e Yay (2015), salientam que o aperfeiçoamento de técnicas de separação de 
resíduos e uma compreensão mais aprofundada sobre as características bioquímicas dos alimentos, podem trazer melhores resultados para aumentar o aproveitamento dos resíduos orgânicos. Os demais autores, deste agrupamento, mencionam formas para melhorar o desempenho da reciclagem do plástico, de resíduos hospitalares, e da construção civil (DAHLBO et al., 2015; RIGAMONTI et al., 2014; WINDFELD; BROOKS, 2015).

No agrupamento Estruturas de destinação final de resíduos, Liu et al. (2014) e Sellin e Leupin (2014), buscam direcionar as suas pesquisas em tópicos que identifiquem os melhores materiais para que os resíduos sejam devidamente acondicionados. Nabavi-Pelesaraei et al. (2017) e Butera, Christensen e Astrup (2015) analisam os materiais que são submetidos a incineração, buscando quantificar os impactos ambientais que são produzidos com essas práticas.

Ferramentas e métodos para gestão de resíduos, agregou diversos procedimentos que são fundamentais para o bom gerenciamento dos resíduos, a começar pela implementação da Avaliação do Ciclo de Vida e da implementação das Normas ISO, para proporcionar subsídios aos gestores públicos, e apoiar na tomada de decisões (LAURENT et al., 2014a, 2014b). Menciona-se também, o apoio de métodos quantitativos, que possa proporcionar aos gestores de resíduos a otimização de processos (SOLTANI et al., 2015; TAN et al., 2014). Kanagaraj et al. (2015) e Tatum, Foy e Aprahamian (2014), se atém às pesquisas mais relacionadas a química em que são destinadas ao desenvolvimento de métodos de biodegradação com resíduos de corantes e processo de hidrólise.

Em Práticas sustentáveis, agrupou-se os artigos que tivessem relação com os três pilares da sustentabilidade: meio ambiente, sociedade e economia (ELKINGTON, 1998). Quanto à questão ambiental, abordou-se o desenvolvimento de tecnologias para baixa emissão de $\mathrm{CO}_{2}$ e análise da biometanização para a gestão de resíduos sólidos urbanos (FERNÁNDEZ-NAVA et al., 2014; YONG et al., 2016). Na parte social, Arena e Gregorio (2014) enfatizaram a importância da inserção de aspectos sociais relacionados ao gerenciamento de resíduos em avaliações do ciclo de vida. Quanto ao aspecto econômico, apontou-se o desenvolvimento de sistemas integrativos de gerenciamento do desperdício de comida em países desenvolvidos e a importância do investimento na coleta de resíduos alimentares para sua utilização como ração animal (SALEMDEEB et al., 2017; THI; KUMAR; LIN, 2015).

\section{Conclusão}

O objetivo de identificar as lacunas científicas existentes sobre o tema foi devidamente alcançado, oportunizando a resposta da questão de pesquisa sobre quais são as principais lacunas científicas e tendências na gestão de resíduos, para a contribuição de um planeta mais sustentável. Os 30 artigos selecionados puderam fornecer subsídios para aclarar as principais oportunidades desse campo do conhecimento, enfatizando a importância e a grande concentração de artigos nas tendências "Ferramentas e métodos para gestão de resíduos" e "Estruturas de coleta e reciclagem".

A principal contribuição deste artigo foi a sistematização da teoria, identificando os grupos Energia renovável; Estruturas de coleta e reciclagem; Estruturas de destinação final de resíduos; Ferramentas e métodos para gestão de resíduos e Práticas sustentáveis. Verificou-se que o empenho acadêmico tem alcançado resultados significativos para o meio ambiente, para sociedade e para a economia.

A principal limitação deste artigo foi não ter publicações mais recentes compondo os 30 mais relevantes, conforme o método utilizado, deixando fora das lentes de pesquisa algumas observações importantes. Como sugestões de estudos futuros, recomenda-se a elaboração de um cenário técnicocientífico dos países mais influentes no gerenciamento de resíduos, servindo como um parâmetro de boas práticas para outras regiões aprimorarem o seu gerenciamento de resíduos sólidos.

\section{Referências}

AGHAJANI MIR, M. et al. Application of TOPSIS and VIKOR improved versions in a multi criteria decision analysis to develop an optimized municipal solid waste management model. Journal of Environmental Management, v. 166, p. 109-115, jan. 2016.

ALLESCH, A.; BRUNNER, P. H. Assessment methods for solid waste management: A literature review. Waste Management \& Research, v. 32, n. 6, p. 461-473, 3 jun. 2014.

ARENA, U.; DI GREGORIO, F. A waste management planning based on substance flow analysis. Resources, Conservation and Recycling, v. 85, p. 54-66, abr. 2014. 
ASSOCIAÇÃO BRASILEIRA DE NORMAS TÉCNICAS. ABNT NBR 10004:2004. Disponível em: <https://www.abntcatalogo.com.br/norma.aspx?ID=936>. Acesso em: 10 jul. 2020.

BRUNNER, P. H.; RECHBERGER, H. Waste to energy - key element for sustainable waste management. Waste Management, v. 37, p. 3-12, mar. 2015.

BUTERA, S.; CHRISTENSEN, T. H.; ASTRUP, T. F. Life cycle assessment of construction and demolition waste management. Waste Management, v. 44, p. 196-205, out. 2015.

CULLEN, J. G. Educating Business Students About Sustainability: A Bibliometric Review of Current Trends and Research Needs. Journal of Business Ethics, v. 145, n. 2, p. 429-439, out. 2017.

DAHLBO, $\mathrm{H}$. et al. Construction and demolition waste management - a holistic evaluation of environmental performance. Journal of Cleaner Production, v. 107, p. 333-341, nov. 2015.

DONG, J. et al. Energy-environment-economy assessment of waste management systems from a life cycle perspective: Model development and case study. Applied Energy, v. 114, p. 400-408, fev. 2014.

ELKINGTON, J. Accounting for the Triple Bottom Line. Measuring Business Excellence, v. 2, n. 3, p. 18-22, mar. 1998.

ERIKSSON, M.; STRID, I.; HANSSON, P.-A. Carbon footprint of food waste management options in the waste hierarchy - a Swedish case study. Journal of Cleaner Production, v. 93, p. 115-125, abr. 2015.

ERSES YAY, A. S. Application of life cycle assessment (LCA) for municipal solid waste management: a case study of Sakarya. Journal of Cleaner Production, v. 94, p. 284-293, maio 2015.

FERNÁNDEZ-NAVA, Y. et al. Life cycle assessment of different municipal solid waste management options: a case study of Asturias (Spain). Journal of Cleaner Production, v. 81, p. 178-189, out. 2014.

$\mathrm{FU}, \mathrm{H}$. et al. A bibliometric analysis of solid waste research during the period 1993-2008. Waste Management, v. 30, n. 12, p. 2410-2417, dez. 2010.

GHIANI, G. et al. Operations research in solid waste management: A survey of strategic and tactical issues. Computers \& Operations Research, v. 44, p. 22-32, abr. 2014.

GUPTA, N.; YADAV, K. K.; KUMAR, V. A review on current status of municipal solid waste management in India. Journal of Environmental Sciences, v. 37, p. 206-217, nov. 2015.

HSIEH, H.-F.; SHANNON, S. E. Three Approaches to Qualitative Content Analysis. Qualitative Health Research, v. 15, n. 9, p. 1277-1288, nov. 2005.

KANAGARAJ, J. et al. Eco-friendly waste management strategies for greener environment towards sustainable development in leather industry: a comprehensive review. Journal of Cleaner Production, v. 89, p. 1-17, fev. 2015.

KOTHARI, C. R.; GARG, G. Research methodology methods and techniques. $4^{\circ} \mathrm{ed}$. Nova Deli: New Age International, 2019.

LAURENT, A. et al. Review of LCA studies of solid waste management systems - Part II: Methodological guidance for a better practice. Waste Management, v. 34, n. 3, p. 589-606, mar. 2014a.

LAURENT, A. et al. Review of LCA studies of solid waste management systems - Part I: Lessons learned and perspectives. Waste Management, v. 34, n. 3, p. 573-588, mar. 2014b.

LIU, H.-C. et al. Site selection in waste management by the VIKOR method using linguistic assessment. Applied Soft Computing, v. 21, p. 453-461, ago. 2014.

MIEZAH, K. et al. Municipal solid waste characterization and quantification as a measure towards 
effective waste management in Ghana. Waste Management, v. 46, p. 15-27, dez. 2015.

MIGUEL, P. A. C. Metodologia de pesquisa para engenharia de produção e gestão de operações. $3^{\circ}$ ed. Rio de Janeiro: Elsevier, 2018.

MILUTINOVIĆ, B. et al. Multi-criteria analysis as a tool for sustainability assessment of a waste management model. Energy, v. 74, p. 190-201, set. 2014.

NABAVI-PELESARAEI, A. et al. Modeling of energy consumption and environmental life cycle assessment for incineration and landfill systems of municipal solid waste management - A case study in Tehran Metropolis of Iran. Journal of Cleaner Production, v. 148, p. 427-440, abr. 2017.

NUNHES, T. V.; OLIVEIRA, O. J. Analysis of Integrated Management Systems research: identifying core themes and trends for future studies. Total Quality Management \& Business Excellence, p. 123, 13 maio 2018.

QAMBRANI, N. A. et al. Biochar properties and eco-friendly applications for climate change mitigation, waste management, and wastewater treatment: A review. Renewable and Sustainable Energy Reviews, v. 79, n. February, p. 255-273, nov. 2017.

RIGAMONTI, L. et al. Environmental evaluation of plastic waste management scenarios. Resources, Conservation and Recycling, v. 85, p. 42-53, abr. 2014.

SALEMDEEB, R. et al. Environmental and health impacts of using food waste as animal feed: a comparative analysis of food waste management options. Journal of Cleaner Production, v. 140, p. 871-880, jan. 2017.

SARATH, P. et al. Mobile phone waste management and recycling: Views and trends. Waste Management, v. 46, p. 536-545, dez. 2015.

SELLIN, P.; LEUPIN, O. X. The Use of Clay as an Engineered Barrier in Radioactive-Waste Management - A Review. Clays and Clay Minerals, v. 61, n. 6, p. 477-498, 1 dez. 2013.

SOLTANI, A. et al. Multiple stakeholders in multi-criteria decision-making in the context of Municipal Solid Waste Management: A review. Waste Management, v. 35, p. 318-328, jan. 2015.

TAN, S. T. et al. Optimal process network for municipal solid waste management in Iskandar Malaysia. Journal of Cleaner Production, v. 71, n. 2014, p. 48-58, maio 2014.

TATUM, L. A.; FOY, J. T.; APRAHAMIAN, I. Waste Management of Chemically Activated Switches: Using a Photoacid To Eliminate Accumulation of Side Products. Journal of the American Chemical Society, v. 136, n. 50, p. 17438-17441, dez. 2014.

THI, N. B. D.; KUMAR, G.; LIN, C.-Y. An overview of food waste management in developing countries: Current status and future perspective. Journal of Environmental Management, v. 157, p. 220-229, jul. 2015.

WILSON, D. C. et al. 'Wasteaware' benchmark indicators for integrated sustainable waste management in cities. Waste Management, v. 35, p. 329-342, jan. 2015.

WINDFELD, E. S.; BROOKS, M. S.-L. Medical waste management - A review. Journal of Environmental Management, v. 163, p. 98-108, nov. 2015.

WORLD BANK GROUP. What a Waste 2.0: A Global Snapshot of Solid Waste Management to 2050.

YONG, J. Y. et al. Cleaner energy for cleaner production: modelling, simulation, optimisation and waste management. Journal of Cleaner Production, v. 111, p. 1-16, jan. 2016.

ZAMAN, A. U. A comprehensive review of the development of zero waste management: lessons learned and guidelines. Journal of Cleaner Production, v. 91, p. 12-25, mar. 2015. 